\title{
Gray-level Texture Characterization Based on a New Adaptive Nonlinear Auto-Regressive Filter
}

\author{
Mounir Sayadi ${ }^{*}$, Samir Sakrani ${ }^{*}$, Farhat Fnaiech $^{*}$, and Mohamed Cheriet ${ }^{+}$ \\ * SICISI Unit, ESSTT, 5 Av. Taha Hussein, 1008, Tunis, Tunisia \\ + École de technologie supérieure, Notre-Dame, Montreal, Quebec H3C 1K3, Canada
}

Received $8^{\text {th }}$ May, 2008; revised $10^{\text {th }}$ November 2008; accepted $26^{\text {th }}$ November, 2008

\begin{abstract}
In this paper, we propose a new nonlinear exponential adaptive two-dimensional (2-D) filter for texture characterization. The filter coefficients are updated with the Least Mean Square (LMS) algorithm. The proposed nonlinear model is used for texture characterization with a 2-D Auto-Regressive (AR) adaptive model. The main advantage of the new nonlinear exponential adaptive 2-D filter is the reduced number of coefficients used to characterize the nonlinear parametric models of images regarding the 2-D second-order Volterra model. Whatever the degree of the non-linearity, the problem results in the same number of coefficients as in the linear case. The characterization efficiency of the proposed exponential model is compared to the one provided by both 2-D linear and Volterra filters and the cooccurrence matrix method. The comparison is based on two criteria usually used to evaluate the features discriminating ability and the class quantification. Extensive experiments proved that the exponential model coefficients give better results in texture discrimination than several other parametric features even in a noisy context.
\end{abstract}

Key words: Image Analysis, 2-D nonlinear filter, 2-D adaptive filter, texture characterization.

\section{Introduction}

The texture analysis plays a significant role in many image processing and pattern recognition applications such as remote sensing, cartography, robot vision, military surveillance and medical imaging. Over the years, many approaches for texture characterization have been developed for analysis [1][35][43] and synthesis [4][5]. The traditional texture characterization is based on extracting some statistic features from the pixel domain data (using histograms, autocorrelation, and moments). In addition, the co-occurrence matrix is a popular statistical technique for extracting textural features. It has been first proposed by [18] for texture characterization and used in the analysis and classification of many types of texture images [16][20]. Furthermore, the texture image was modeled in several techniques as a Markov random field of pixels gray level, where the relationships between the gray level of neighboring pixels are statistically characterized [35][45]. In [35], a probabilistic texture model using a Gauss-Markov random field has been proposed for hyperspectral textures characterization. Also, multi-resolution models for Gauss-Markov random fields with applications to texture characterization were proposed in [21] and applied for texture segmentation.

In spite of this, feature-based approaches have been proposed during the last two decades which is sometimes less computationally demanding and more effective than Markov random field based approaches. Textural features are typically extracted by using spectral information [35], wavelet basis functions [42] and Gabor filters [1][7]. The 2-D Gabor filters have been proved to be an important tool in texture analysis. They consist of a sinusoidal plane wave of some frequency and orientation modulated by Gaussian envelope. A Gabor filter is a band-pass filter which can be used to extract a specific band of frequency components from an image [27]. Gabor functions appear to share many properties with the human visual system [25]. They are used as non-orthogonal basis functions for signal representation their magnitude responses in the frequency domain is well-behaved, having no side lobes [16]. 
In [13], the authors compare several texture features based on the local power spectrum obtained by a bank of Gabor filters. These features are: Gabor energy, complex moments, and grating cell operator features. It has been shown that the grating cell operator gives the best discrimination and segmentation results. More recently, Gabor filters and gray level co-occurrence probability features have been fused in [7] to improve the texture recognition and segmentation. It has been also shown in [1] that for noisy texture recognition, the features based on Gabor filters outperform the Discrete Fourier Transform features. In addition, steerable pyramid model has been proposed by [12] as a rotation invariant texture model. It can be provided by the discrete Fourier transform of a feature curve defined across the orientation space of the texture. Also, a rotation-invariant image retrieval scheme based on a transformation of the texture information via a steerable pyramid has been also presented in [41] where the distributions of the subband coefficients are exploited to capture their non-Gaussian behavior. Then, a normalization process in order to Gaussianize the coefficients is applied [41]. More recently, a new rotation and scale invariant representations for texture image retrieval based on steerable pyramid decomposition have been proposed in [28]. In this approach, the texture feature vectors are extracted by calculating the mean and standard deviation of decomposed image subbands [28].

Moreover, the Local Binary Pattern (LBP) method has been proposed for texture characterization in [29] and [30]. In this method, a local window pattern from the texture is coded, and its histogram is often considered as textural feature. In [31], an efficient, multiresolution approach to gray-scale and rotation invariant texture classification based on LBP and non-parametric discrimination of sample and prototype distributions. In [44], a promising method of texture segmentation based on the LBP histogram energy function has been proposed. More recently, an algorithm based on the LBP was proposed in [43] where the central pixel in the local region was added into the procedure to calculate the local threshold using a modified version of the Niblack algorithm.

In addition, a multispectral texture segmentation method based on a causal adaptive regression model prediction was proposed in [15]. Also, the authors in [34] reviewed several filtering approaches of texture feature extraction such as Laws masks, ring-wedge filters, wavelet transforms, quadrature mirror filters, discrete cosine transform, eigen-filters, Gabor filters and linear predictor filters. These methods have been compared to the co-occurrence and AR model based methods. Furthermore, it is observed that some secondorder non-parametric methods, such as spectrum estimation [34][36] are not capable of significantly characterizing textures that have quite different visual characteristics specially when only small sample texture images are accessible, while higher order methods [37][38] have a high computational cost. More recently, a novel approach for texture characterization and synthesis based on Textons (texture elements) was recently proposed in [4] where textures are assumed to be composed of three components, namely illumination, structure, and stochastic. Also, the authors in [10][11] have proposed a unified texture model based on 2-D Wold-like decomposition. This model is appropriate for modeling real-world textures and describing its randomness. The texture is decomposed into three mutually orthogonal components: stochastic, harmonic and generalized-evanescent component [11]. Different methods of estimation of these three components were proposed [10].

In the present paper, we address the estimation of only one part of the 2-D Wold model of gray-scale textures, namely the 2-D stochastic component. This component can be represented by a 2-D Auto-Regressive (2-D AR) parametric model [5][24][36]. This model, commonly used in signal processing, offers an expression of the spatial interaction among the neighbors pixel in the image [5][6]. In [6], a promising texture segmentation algorithm based on a 2-D AR linear model with an adapted neighborhood and a multi-scale approach was presented. In [5], the authors proposed a 2-D AR model to characterize the gray level at a pixel as a linear combination of gray levels of its neighbor's pixels. Generally, the parametric model can be estimated by using existing estimation methods of purely stochastic random fields. An alternative method for the parametric model computing is to employ adaptive algorithms to obtain the estimate of the observed field model [14][37][38]. In spite of this, the linear adaptive parametric filters fail in many 2-D applications, such as image enhancement, image modeling and compression because of the nonlinear and non stationary usual character of images to be processed. Furthermore, experimental results indicate that some processing levels of the visual system have nonlinear characteristics [32].

Looking for better results, many researchers focus their attention on nonlinear adaptive filtering [23][26][33]. In [23], adaptive 2-D Volterra filters have been applied to nonlinear channel distorted image restoration. Since the nonlinear adaptive parametric filters use a high number of coefficients to represent the image nonlinear character, research of models providing low computation cost is an appealing subject. 
In the present paper, we propose a new nonlinear model for 2-D signal modeling which takes into consideration a high order non-linearity without increasing the number of filter coefficients. We propose to use the exponential of the 2-D support matrix instead of using the support matrix itself. Consequently, whatever the degree of the non-linearity is, the problem results in the same number of coefficients as in the linear case. Furthermore, the proposed 2-D AR exponential filter is applied for gray-level texture characterization. We illustrate how much this model improves the gray-level texture classification in comparison to the linear model [14], the 2-D Volterra model [23] and the co-occurrence matrix method [18]. For such a purpose, we evaluate two characterization criteria conventionally used in the pattern recognition field. The first is a classification accuracy based on a weighted Euclidean distance classifier. It was used by many authors to test the classification robustness of several features. The second is a characterization degree based on the ratio between the "inter-variance" and the "intra-variance" of the coefficients. The greater the characterization degree is, the more robust the classification process is.

The initial idea of the exponential model has been presented in [39]. Meanwhile, we propose in the present paper an in-depth version containing the following additional items: a comparison of the proposed method to the second-order 2-D Volterra filter, a study of the coefficient number and an additional classification criterion based on a weighted Euclidean distance classifier. Furthermore, more textures are studied.

\section{Recall of the 2-D linear model and the LMS algorithm}

An image $y$ of size $(L \times L)$ can be represented by a 2-D transversal AR parametric linear model with a noncausal square support of order $(p \times p)$ as schematized in Figure 1. The value of an image pixel at the $n^{\text {th }}$ column and the $r^{\text {th }}$ line is represented by the following linear relationship:

$$
y(n, r)=\sum_{i=-\frac{p-1}{2}}^{\frac{p-1}{2}} \sum_{j=-\frac{p-1}{2}}^{\frac{p-1}{2}}{ }_{(i, j) \neq(0,0)} w(i, j) y(n+i, r+j)
$$

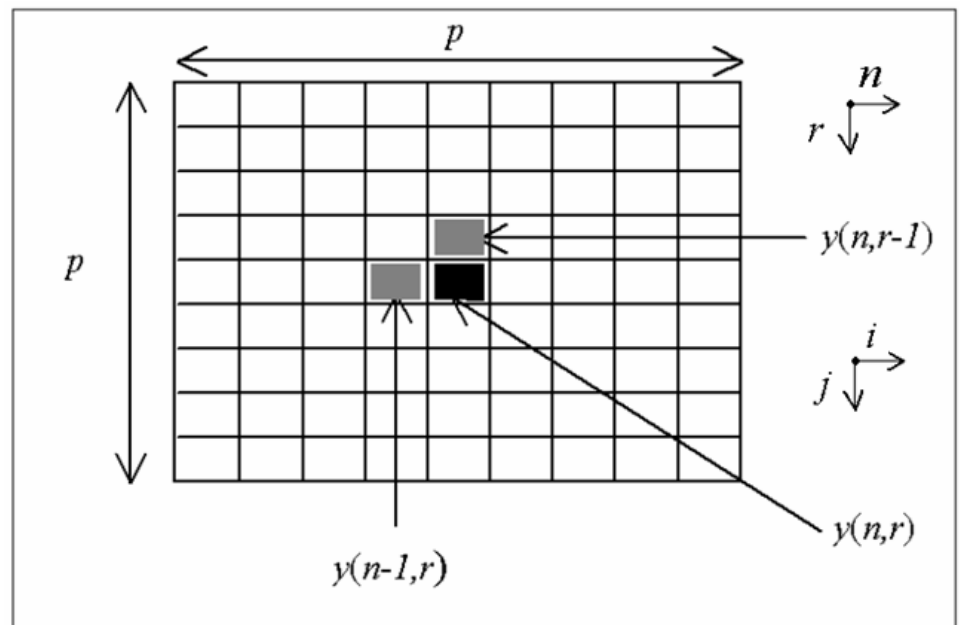

Figure 1: Two-dimensional filter support

Our aim for choosing a square order is to simplify the extension of the linear model to the 2-D nonlinear exponential model. The filter order $p$ is supposed of odd values. $n$ and $r$ are in the interval $[1 . . L]$, and $w(i, j)$ are the 2-D AR linear filter coefficients. In the stationary case, these coefficients do not depend on the position of the pixel $(n, r)$. The form (1) of the filter output can be rewritten by:

$$
y(n, r)=Y(n, r) * W
$$

where $W$ denotes a matrix formed by the filter coefficients as: 


$$
W=\left(\begin{array}{cccc}
w\left(-\frac{p-1}{2},-\frac{p-1}{2}\right) & w\left(-\frac{p-1}{2}+1,-\frac{p-1}{2}+1\right) & \cdots & w\left(-\frac{p-1}{2}, \frac{p-1}{2}\right) \\
w\left(-\frac{p-1}{2}+1,-\frac{p-1}{2}\right) & w\left(-\frac{p-1}{2}+1,-\frac{p-1}{2}+1\right) & \cdots & w\left(-\frac{p-1}{2}+1, \frac{p-1}{2}\right) \\
\vdots & \vdots & \ddots & \vdots \\
w\left(\frac{p-1}{2},-\frac{p-1}{2}\right) & w\left(\frac{p-1}{2},-\frac{p-1}{2}+1\right) & \cdots & w\left(\frac{p-1}{2}, \frac{p-1}{2}\right)
\end{array}\right),
$$

and $Y(n, r)$ denotes the matrix window containing the gray level of pixels enclosed by the bi-dimensional filter support as follows:

$$
Y(n, r)=\left(\begin{array}{llll}
y\left(n-\frac{p-1}{2}, r-\frac{p-1}{2}\right) & y\left(n-\frac{p-1}{2}, r-\frac{p-1}{2}+1\right) & \cdots & y\left(n-\frac{p-1}{2}, r+\frac{p-1}{2}\right) \\
y\left(n-\frac{p-1}{2}+1, r-\frac{p-1}{2}\right) & y\left(n-\frac{p-1}{2}+1, r-\frac{p-1}{2}+1\right) & \cdots & y\left(n-\frac{p-1}{2}+1, r+\frac{p-1}{2}\right) \\
\vdots & \vdots & \ddots & \vdots \\
y\left(n+\frac{p-1}{2}, r-\frac{p-1}{2}\right) & y\left(n+\frac{p-1}{2}, r-\frac{p-1}{2}+1\right) & \cdots & y\left(n+\frac{p-1}{2}, r+\frac{p-1}{2}\right)
\end{array}\right)
$$

and '.*' denotes the dot product of $Y(n, r)$ and $W$.

In order to update the filter coefficients, the adaptive LMS algorithm [14][40] is the most widely used algorithm due to its simple implementation. It is based on minimizing the instantaneous square error $J$ between the filter output $y(n, r)$ and the desired output $d(n, r)$. This square error is defined by:

$$
J=\xi^{2}(n, r)=(d(n, r)-y(n, r))^{2} .
$$

The gray level value of the texture pixel is noted $d(n, r)$. The filter error $d(n, r)-y(n, r)$ is noted $\xi(n, r)$. The adaptation of the coefficients matrix $W$ of equations and (3) with the 2-D LMS algorithm [10][31] can be given by: $W^{(k+1)}=W^{(k)}-\frac{1}{2} \mu \frac{\partial J}{\partial W^{(k)}}$.

In this expression, $\mu$ is the step size of the algorithm. The spatial scanning order of the image of size $(L \times L)$ is performed, as shown in Figure 2, from left to right and top to bottom by means of a linear scanning index $k=n L+r$.

The matrix $W^{(k)}$ denotes the matrix of the filter coefficients of equation (3) at time $k$. The gradient of $J$ with respect to the coefficients matrix $W^{(k)}$ is: $\frac{\partial J}{\partial W^{(k)}}=-2 \xi(n, r) \frac{\partial y(n, r)}{\partial W^{(k)}} \approx-2 \xi(n, r) Y(n, r)$.

The last approximation (7) have been proved in [9] for auto-regressive models and do not cause a significant maladjustment. Hence the adaptation equation can be written as:

$$
W^{(k+1)}=W^{(k)}+\mu \xi(n, r) Y(n, r),
$$

which can be also written in an explicit form with:

$$
\begin{gathered}
\text { For } i \text { from }-\frac{p-1}{2} \text { to } \frac{p-1}{2}, \quad \text { For } j \text { from }-\frac{p-1}{2} \text { to } \frac{p-1}{2} \\
w^{(k+1)}(i, j)=w^{(k)}(i, j)+\mu(d(n, r)-y(n, r)) y(n+i, r+j) .
\end{gathered}
$$

The initial values of the coefficients matrix $W^{(0)}$ can be set to zero matrix. The equation (1) is interpreted graphically in Figures 2 and 3. To produce an approximate value of $y(n, r)$, all the values of the pixel image which are covered by the mask (i.e. the filter support), except the one at $(n, r)$, weighted by the coefficients $w^{(k)}(i, j)$ and summed. After the adaptation of the matrix coefficients $W^{(k)}$, the mask is then moved to the following location $(n+1, r)$. 


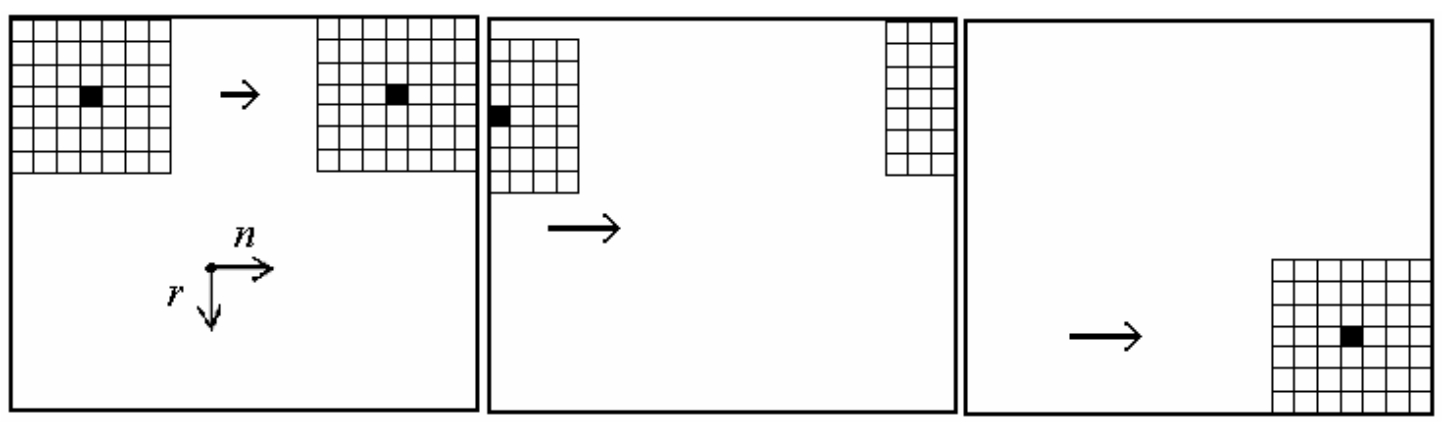

Figure 2: The adaptive scanning mask from left to right and top to bottom

Texture to be modelized

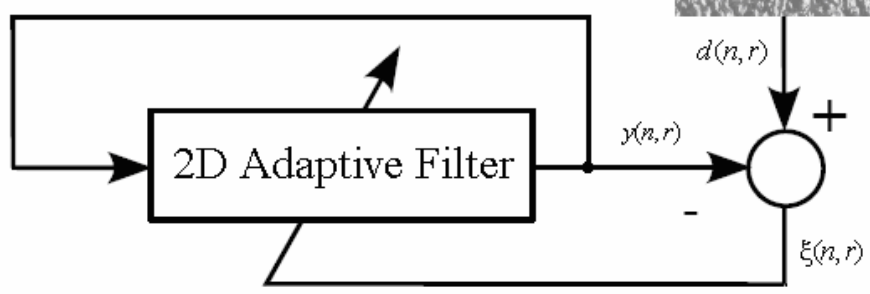

Figure 3: Block diagram of the principle of the 2-D AR adaptive filtering of a texture

\section{The 2-D second-order Volterra model}

The 2-D nonlinear modeling is more accurate than the linear modeling in image filtering to take into account the nonlinear character of some images. The second-order Volterra model is the most popular nonlinear filter used in 1-D context [40]. Hence, it has been extended to 2-D signals [23] and it was used for 2-D nonlinear channel equalization and image restoration with good results. Considering the filter support of Figure 1, the value of a pixel at position $(n, r)$ can be now expressed by a second-order Volterra model as:

$$
\begin{aligned}
y(n, r)= & \sum_{i=-\frac{p-1}{2}}^{\frac{p-1}{2}} \sum_{j=-\frac{p-1}{2}}^{\frac{p-1}{2}} \\
& +\sum_{i_{1}=-\frac{p-1}{2}}^{\frac{p-1}{2}} \sum_{j_{1}=-\frac{p-1}{2}}^{\frac{p-1}{2}} \sum_{i_{2}=-\frac{p-1}{2}}^{\frac{p-1}{2}} \sum_{j_{2}=-\frac{p-1}{2}(0,0)}^{\frac{p-1}{2}} w_{L}(i, j) y(n+i, r+j) \\
\left(\begin{array}{l}
\left(i_{1}, j_{1}\right) \neq\left(j_{2}\right) \neq(0,0) \\
2
\end{array}\right. & w_{Q}\left(i_{1}, j_{1}, i_{2}, j_{2}\right) y\left(n+i_{1}, r+j_{1}\right) y\left(n+i_{2}, r+j_{2}\right),
\end{aligned}
$$

$w_{L}(i, j)$ and $w_{Q}\left(i_{1}, j_{1}, i_{2}, j_{2}\right)$ are respectively the linear and the quadratic filter coefficients.

The derivation of the 2-D LMS algorithm for this model is performed by minimizing the cost function (5). As has been demonstrated in [40], the error $J$, given in (5) for only the linear filter, is now the sum of two errors corresponding respectively to the linear and quadratic filter parts.

The gradient of $J$ to be minimized is then the sum of $\frac{\partial J}{\partial W_{L}^{(k)}}$ and $\frac{\partial J}{\partial W_{Q}^{(k)}}$ where the matrix $W_{L}^{(k)}$ and $W_{Q}^{(k)}$ respectively denote the matrix containing the linear and the quadratic Volterra coefficients at time $k$.

The first gradient part is calculated for each linear coefficient as: 


$$
\frac{\partial J}{\partial W_{L}^{(k)}}=2 \xi(n, r) \frac{\partial \xi(n, r)}{\partial W_{L}^{(k)}}=-2 \xi(n, r) \frac{\partial y(n, r)}{\partial W_{L}^{(k)}} \approx-2 \xi(n, r) y(n+i, r+j)
$$

This expression will be used to adapt the linear filter coefficients.

For the nonlinear filter coefficients, the gradient part is calculated for each coefficient as:

$$
\frac{\partial J}{\partial W_{Q}^{(k)}}=2 \xi(n, r) \frac{\partial \xi(n, r)}{\partial W_{Q}^{(k)}}=-2 \xi(n, r) \frac{\partial y(n, r)}{\partial W_{Q}^{(k)}} \approx-2 \xi(n, r) y\left(n+i_{1}, r+j_{1}\right) y\left(n+i_{2}, r+j_{2}\right) .
$$

Thus, the Volterra coefficients updated with the2-D LMS algorithm can be summarized by:

$$
\begin{aligned}
& w_{L}^{(k+1)}(i, j)=w_{L}^{(k)}(i, j)+\mu_{1} \xi(n, r) y(n+i, r+j) \\
& w_{Q}^{(k+1)}\left(i_{1}, j_{1}, i_{2}, j_{2}\right)=w_{Q}^{(k)}\left(i_{1}, j_{1}, i_{2}, j_{2}\right)+\mu_{2} \xi(n, r) y\left(n+i_{1}, r+j_{1}\right) y\left(n+i_{2}, r+j_{2}\right)
\end{aligned}
$$

In these expressions $\mu_{1}$ and $\mu_{2}$ are the step sizes of the algorithm.

Although the Volterra nonlinear filter may be more accurate than the linear filter for image modeling, it has a significant drawback i.e. the giant increase in the number of coefficients when large support size is to be considered, as seen in Table 1. To overcome this inconvenient, we propose in the following an exponential model as a new nonlinear model for texture characterization.

\section{The Proposed exponential model and its adaptation with the LMS algorithm}

In the proposed nonlinear filter, the filter output $y(n, r)$ is calculated by using the exponential matrix of the 2-D support. For this, we define the matrix $E y(n, r)$ as the exponential of the matrix $Y(n, r)$ given by :

$$
\operatorname{Ey}(n, r)=\operatorname{Exp}(Y(n, y))-I_{p \times p}
$$

$\operatorname{Exp}(Y(n, y))$ is the exponential of the matrix $Y . I_{p \times p}$ is the $p \times p$ identity matrix.

The output of the proposed exponential filter is then given by:

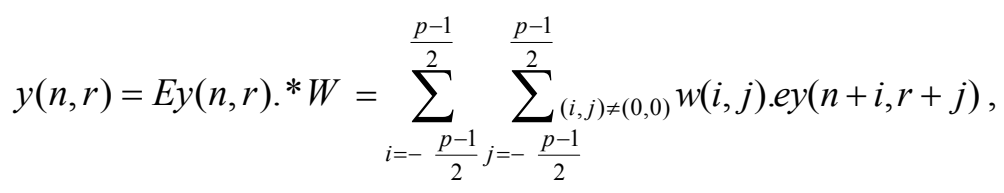

where $e y(n+i, r+j)$ is the $(i, j)$ element of the matrix $E y(n, r)$.

The 2-D LMS algorithm can be used for adapting the exponential filter by minimizing the square error $J$ between the filter output expressed by (16) and the desired output $d(n, r)$.

$$
J=\xi^{2}(n, r)=(d(n, r)-y(n, r))^{2}
$$

The gradient of $J$ with respect to the matrix coefficients is simply calculated in the same way than in the linear case: $\frac{\partial J}{\partial W^{(k)}}=2 \xi(n, r) \frac{\partial \xi(n, r)}{\partial W^{(k)}}=-2 \xi(n, r) \frac{\partial y(n, r)}{\partial W^{(k)}} \approx-2 \xi(n, r) E y(n, r)$.

Hence the adaptation equation is: $W^{(k+1)}=W^{(k)}-\frac{1}{2} \mu \frac{\partial J}{\partial W^{(k)}}=W^{(k)}+\mu \xi(n, r) E y(n, r)$.

Then the 2-D LMS algorithm for the proposed exponential filter can be summarized as follows:

For $n$ and $r$ from 1 to $L$ :

- Calculation of the adaptive filter output by using (16).

- Adaptation of the coefficients: For $i$ and $j$ from $-\frac{p-1}{2}$ to $\frac{p-1}{2}$, 


$$
w^{(k+1)}(i, j)=w^{(k)}(i, j)+\mu(d(n, r)-y(n, r)) e y(n+i, r+j)
$$

Note that the matrix $W^{(k)}$ contains both linear and nonlinear coefficients of the exponential filter. Clearly, the exponential filter model is simpler than the second-order Volterra model. In addition, the number of coefficients remains roughly the same as in 2-D linear filter, whereas the numbers of coefficients of the Volterra filter increases in an exponential manner with respect to the filter order. Table 1 gives the number of coefficients for linear, exponential and second-order Volterra filter models, where $p \times p$ is the dimension of the square sliding window is.

\begin{tabular}{|c|c|c|c|c|c|c|}
\cline { 3 - 7 } \multicolumn{2}{c|}{} & \multicolumn{5}{c|}{ Exemples } \\
\hline Filter model of order $p \times p$ & Number of coefficients & $p=3$ & $p=5$ & $p=7$ & $p=9$ & $P=11$ \\
\hline Linear model & $p^{2}-1$ & 8 & 24 & 48 & 80 & 120 \\
\hline Exponential model & $p^{2}$ & 9 & 25 & 49 & 81 & 121 \\
\hline Second-order Volterra model & $\left(p^{2}-1\right)^{2}+p^{2}-1$ & 72 & 600 & 2352 & 6480 & 14520 \\
\hline
\end{tabular}

Table 1: Number of coefficients for the three used models.

\section{Experimental results on texture characterization based on the Euclidian distance}

Consider a set of 25 different gray-scale textures of $256 \times 256$ pixels (Table 2) extracted from the Brodatz album [1]. In order to check whether the use of the 2-D nonlinear exponential filter improves the texture classification in comparison to the 2-D linear filter and the second-order Volterra filter, we propose to evaluate the characterization efficiency of the coefficients estimated by each filter. For each texture image, a set of 100 randomly chosen images of $(64 \times 64)$ pixels is divided into two non-overlapping sets of 50 sample images. The first set is used to build the reference set for the texture classification experiment. The second set is the testing set. So the reference and the testing sets are separable since they contain non-overlapping blocks. For each of the 2500 resulting images, the 2-D exponential adaptive filter is applied to provide a matrix of estimated coefficients. The filter desired output $d(n, r)$ is the image texture itself. For comparison reason, the 2D linear coefficients and 2-D Volterra coefficients, respectively detailed in section 2 and 3, are also evaluated for all texture images. These three families of estimated coefficients are used as features for the texture classification procedure detailed in the following.

\section{Experiment 1: Effect of the filter order on the texture classification}

The aim of the first experiment is to compare the capability of the three studied parametric adaptive filters i.e. the linear, exponential and Volterra 2-D filters in texture classification for various 2-D filter order. In this experiment, a minimum distance classifier using the class mean and variance is used as a comparison criterion.

For each texture coefficient vector of index $k$, chosen from the testing set, the weighted Euclidian distances are measured between the vector and the mean of the coefficient vector of each of the 25 textures classes belonging to the reference set. Note $\underline{x}_{k, n}$ the $n^{\text {th }}$ estimated coefficient vector for the $k^{\text {th }}$ texture class, (chosen from the reference set), $(1 \leq k \leq 25,1 \leq n \leq 50)$. The means of the $k^{\text {th }}$ texture class coefficient vectors are noted [19]:

$$
\underline{m}_{k}=\frac{1}{50} \sum_{n=1}^{50} \underline{x}_{k, n} .
$$

The variances of the vectors are noted $\underline{v}_{k}=\frac{1}{50} \sum_{n=1}^{50}\left(\underline{x}_{k, n}-\underline{m}_{k}\right)^{2}$. 
Similarly, the testing set is composed by 50 other images for each texture class. Note $y_{i, j}$ the $j^{\text {th }}$ estimated coefficient vector for the $i^{\text {th }}$ texture class $(1 \leq i \leq 25,1 \leq j \leq 50)$. The weighted Euclidian distance measured between the coefficients vector $y_{i, j}$ (chosen from the testing set) and the mean of the coefficient vector $\underline{m}_{k}$

(of the reference set) is given by the distance: $\operatorname{dist}\left(y_{i, j}, \underline{m}_{k}\right)=\sum_{t=1}^{n c} \frac{\left(y_{i, j}(t)-\underline{m}_{k}(t)\right)^{2}}{\underline{v}_{k}(t)}$,

where $n c$ is the total number of coefficients in the vector and $t$ an index $(1 \leq t \leq n c)$. Note that the greater the "within-class" variance of coefficients $\underline{v}_{k}$ is, the smallest the distance $\operatorname{dist}\left(y_{i, j}, \underline{m}_{k}\right)$ is. So, each texture image of the testing set will be assigned with the index $k$ of the texture for which this distance is minimal. For each texture class $i$, we define the classification accuracy as the ratio of the number of positive tests $N_{p t}$ to the total number of tests, i.e. $C A_{i}=\frac{N p t}{50}$. A total classification accuracy $T C$ is calculated for each coefficients family by the mean $T C=\frac{1}{25} \sum_{i=1}^{25} C A_{i}$.

Hence, as stated above, we use the total classification accuracy $T C$, given by (24), as a comparison criterion. This accuracy is calculated for various filter orders ranging from $(3 \times 3)$ to $(13 \times 13)$ without any additive noise. Figure 4 depicts the classification accuracy with respect to the 2-D filter orders.

The step size is equal to 0.1 for all filters. This value is chosen experimentally as the biggest value which guaranties the stability of the LMS algorithm. The size of the sample images is $(64 \times 64)$ pixels. The features extracted from the co-occurrence features [42] are also tested. The elements of the co-occurrence matrix $C_{i, j}$ represents how often pairs of pixels with values $i$ and $j$ separated by a distance $d$ occurs (we have used $d=1$ ). Four directions were used to obtain the co-occurrence matrices (horizontal, first diagonal, vertical and second diagonal i.e. $0^{\circ}, 45^{\circ}, 90^{\circ}$ and $135^{\circ}$ ). In our experimental results, we have chosen the direction which gives the best classification accuracy. A set of seven standard features are extracted from these co-occurrence matrices for each studied image. These features are: the contrast, the energy, the entropy, the homogeneity, the maximum probability, the cluster shade and the cluster prominence [42].

In conclusion, it should be noted that for any order, the classification accuracy provided by the exponential adaptive filter is greater than the one provided by the other filters. Furthermore, the coefficients of the exponential filter seem to give the best classification accuracy for a filter order of $(9 \times 9)$. The classification accuracy is there around 98.4 percent. For greater filter orders, we notice that the classification accuracy decreases. This can be explained by the fact that the size of the filtered texture images $(64 \times 64$ pixels $)$ is not enough for the convergence of the 2-D LMS filters. In addition, although the second-order Volterra and the exponential filter theoretically perform identically, the exponential model is truncated to the order 3 . With this order, the exponential model contains more "nonlinearity order" than the second-order Volterra model and uses fewer coefficients. In addition, we note that the classification accuracy of the co-occurrence features is constant with respect to the filter order. The co-occurrence based method does not use a parametric filter.

Furthermore, the classification accuracy of the exponential filter is larger than those given by the secondorder Volterra filter. We think that the high correlation in the nonlinear expression of the Volterra filter may cause a decrease in the texture characterization robustness by including some redundant information. Finally, to have an idea of the resemblance between the exponential filter coefficients inside the same type of texture, we give in Table 3 the values of two coefficients $\left(w_{00}\right.$ and $\left.w_{01}\right)$ estimated from 3 randomly chosen images from Texture 1 and Texture 2 of Table 2. We note that the coefficients of each texture are grouped together and relatively spread far from the other texture coefficients, which is suitable in the classification process. 


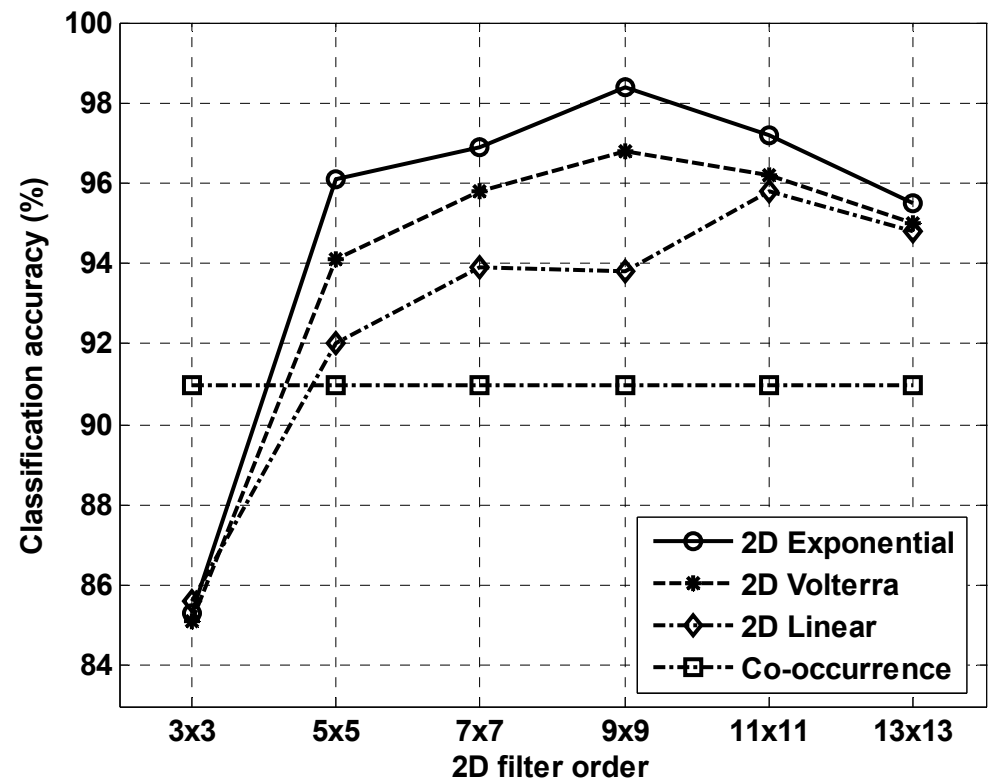

Figure 4: Plot of the classification accuracy in \% of the 25 textures by using of the 2-D adaptive exponential, Volterra and linear filters as well as the co-occurrence method with respect to the filter orders.

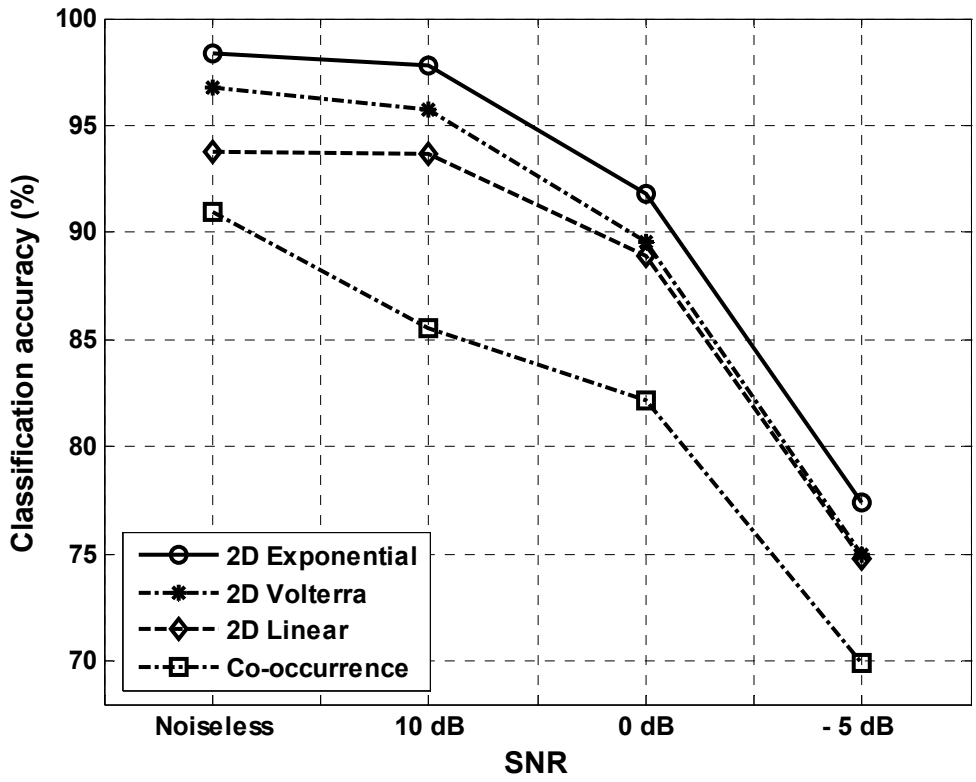

Figure 5: Plot of the classification accuracy in $\%$ of the 2-D adaptive exponential, Volterra and linear filters as well as the co-occurrence method for various SNR values (Filter order $9 \times 9$ ). 


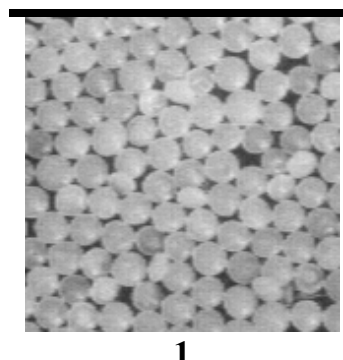

1

Bulle (D66)

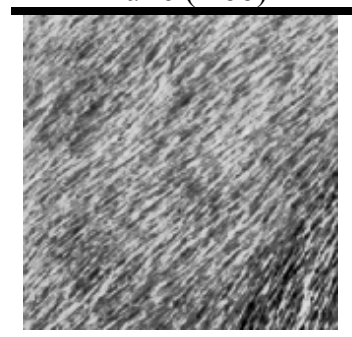

6

Fur (D93)

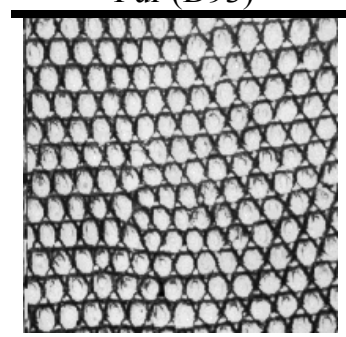

11

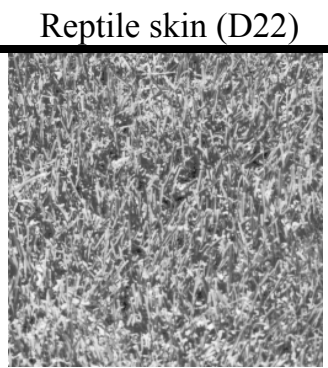

16

Grass (D9)

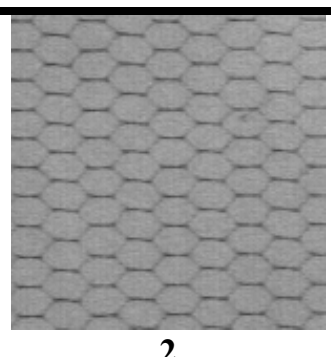

Netting (D34)

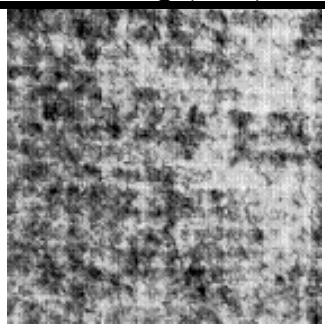

7

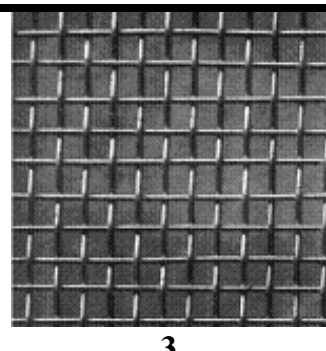

3

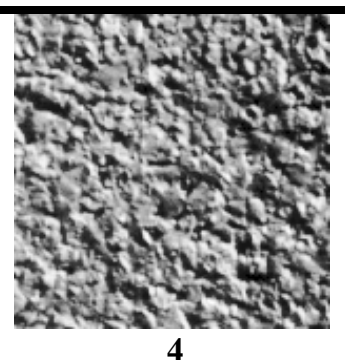

Pressed cork (D4)

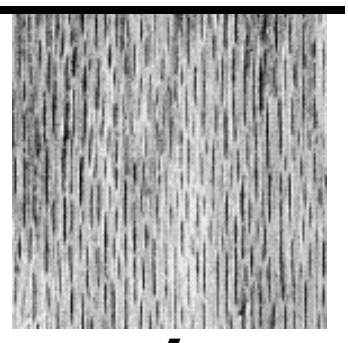

5

Wood (D68)

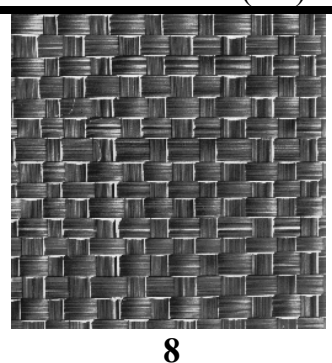

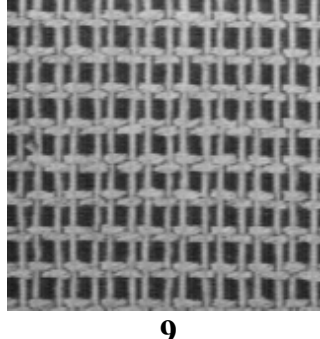

French canvas (D20)

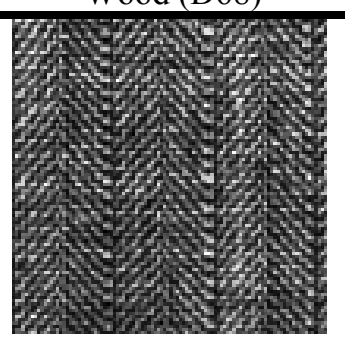

10

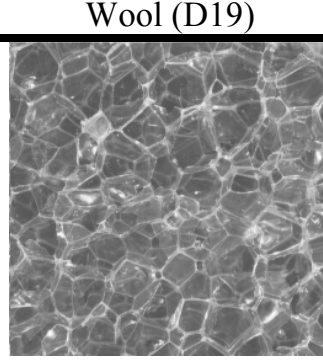

12

Bubbles (D112)

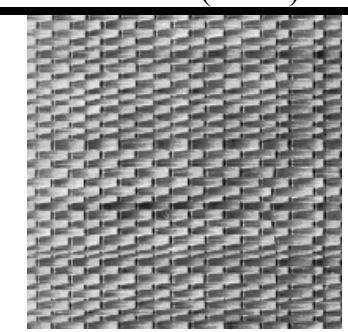

17

Straw matting (D55)

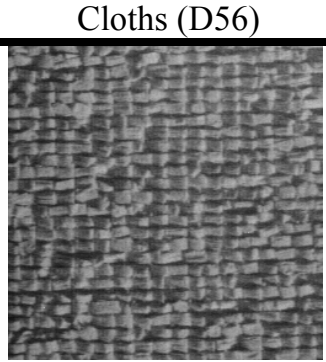

13

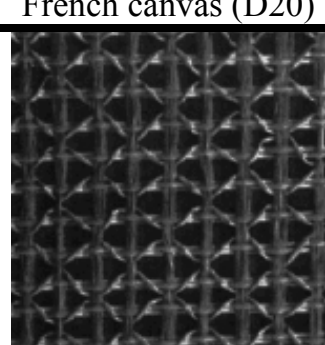

14
Woollen cloth (D11)

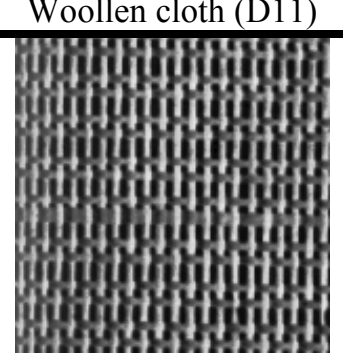

15
Raffia (D84)

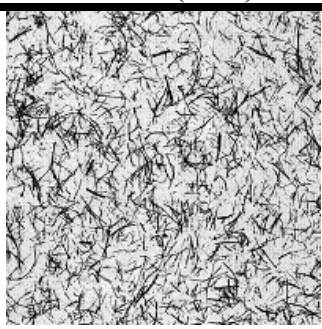

18

Oriental cloth (D52)

Oriental cloth (D53)

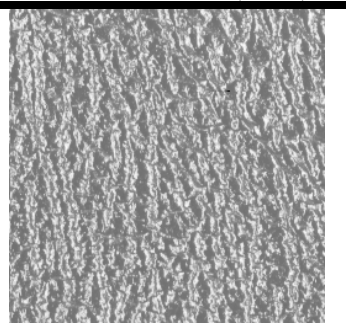

19

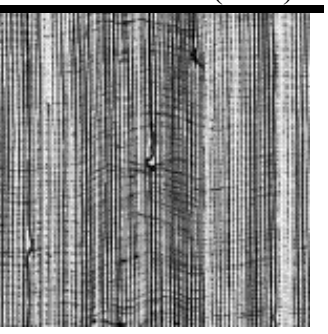

20

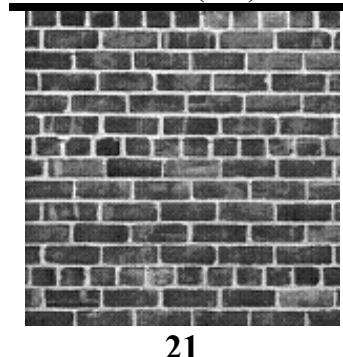

Brick wall (D95)

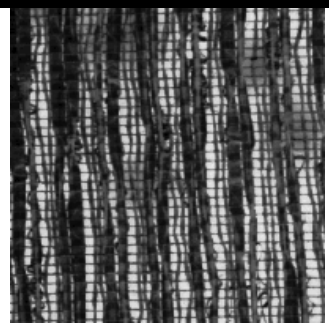

22

Random fibber (D110)

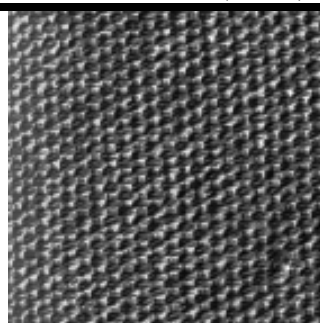

23

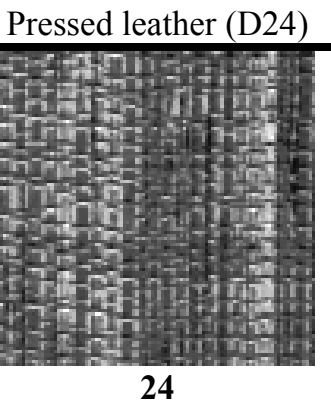

24

Cheese cloth (D106)

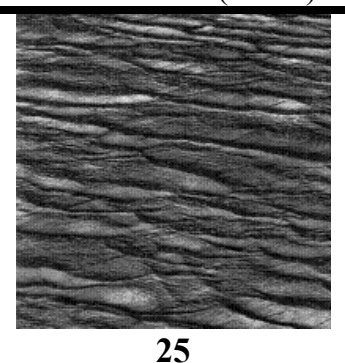

Water (D37)

Table 2: The 25 Brodatz textures used in the study

\begin{tabular}{|c|c|c|c|c|c|c|}
\hline & & Texture 1 & & & Texture 2 & \\
\hline & Image 1 & Image 2 & Image 3 & Image 1 & Image 2 & Image 3 \\
\hline$w_{00}$ & 0.244 & 0.229 & 0.221 & 0.298 & 0.288 & 0.305 \\
\hline$w_{01}$ & 0.307 & 0.314 & 0.321 & 0.214 & 0.224 & 0.201 \\
\hline
\end{tabular}

Table 3: Values of 2 coefficients of the 2-D exponential filter estimated from 3 sample images from the Texture 1 and the Texture 2 . (Filter order $5 \times 5$ ) 


\section{Experiment 2: Effect of the additive noise on the classification accuracy}

In this experiment, we study the effect of a Gaussian additive noise on the texture classification with an exponential adaptive filter. According to the results of the experiment 1 , a filter order of $(9 \times 9)$ gives the better classification accuracy for the studied nonlinear filters, so we have chosen in this experiment to use a filter order of $(9 \times 9)$. For comparison reason, the 2-D Volterra coefficients, the 2-D linear filter coefficients and the co-occurrence features [42] are also evaluated for all texture images.

The obtained four families of estimated coefficients are used as features for the texture classification procedure. The results of this experiment are given in Figure 5 where we plot the classification accuracy $T C$ (24) in percent for all the 25 texture classes with respect to the Signal to Noise Ratio (SNR) value of the additive noise ranging from $10 \mathrm{~dB}$ to $-5 \mathrm{~dB}$. The results show that the coefficients of the 2-D nonlinear exponential filter perform better than those of the other features, and this for all SNR values. Furthermore, the increase of the additive noise variance causes a large attenuation in the classification rate. The additive noise perturbs the classification process.

\section{Experiment 3: Study of the characterization degree in presence of additive noise}

For an in-depth study of the characterization capability of the proposed features, we compute a "characterization degree" $D$ based on the ratio between the "inter-variance" and the "intra-variance" of each feature family classes [19]. Unlike the classification experiment, all the 100 randomly chosen images are used for each texture image. Note $\underline{x}_{k, n}$ the $n^{\text {th }}$ estimated vector of coefficients for the $k^{\text {th }}$ texture class $(1 \leq k \leq 25$, $1 \leq n \leq 100)$. The mean of the $k^{\text {th }}$ texture class vectors of coefficient is noted $\underline{m}_{k}=\frac{1}{100} \sum_{n=1}^{100} \underline{x}_{k, n}$ and the mean of all the coefficient vectors is $\underline{m}_{c}=\frac{1}{25} \sum_{k=1}^{25} \underline{m}_{k}$. The mean of the within-class (intra-class) dispersion matrices is given by the matrix $S_{\text {intra }}=\frac{1}{2500} \sum_{k=1}^{25} \sum_{n=1}^{100}\left(\underline{x}_{k, n}-\underline{m}_{k}\right)\left(\underline{x}_{k, n}-\underline{m}_{k}\right)^{t}$, which is the maximum likelihood estimation of the covariance matrix of the class. Complementary to this is the mean of the between-class (inter-class) dispersion matrices which describes the scattering of the class sample means. It is calculated by the matrix $S_{\text {inter }}=\frac{1}{25} \sum_{k=1}^{25}\left(\underline{m}_{k}-\underline{m}_{c}\right)\left(\underline{m}_{k}-\underline{m}_{c}\right)^{t}$. So, the "characterization degree" $C D$ is given by [19]:

$$
C D=\operatorname{trace}\left(S_{\text {intra }}^{-1} \cdot S_{\text {inter }}\right) \text {. }
$$

The greater this characterization degree is, the more robust the classification process is. The comparison of the ability of the studied features in presence of additive noise will be presented through Figure 6 where we plot the Characterization Degree $C D$ (25) calculated with the features issued from the exponential filter, the 2D Volterra filter, the 2-D linear filter and the co-occurrence method. For the last method, we have chosen the direction which gives the best characterization degree. Four cases are considered: the noiseless case, and three noisy cases of SNR values: $10 \mathrm{~dB}, 0 \mathrm{~dB}$ and $-5 \mathrm{~dB}$, respectively. An additive Gaussian noise and a 2-D order of $9 \times 9$ have been used. We notice that the characterization degree provided by the 2-D exponential filter coefficients is greater than the characterization degrees provided by the other coefficient families. For all cases, the characterization degree decreases with the increase of the noise variance. In addition, we note that for uniformly distributed additive noise, we obtain similar results of the characterization degree. So, as well as the 2-D exponential filter provides the best results in the texture classification experiment, this experiment shows their superiority for texture characterization. 


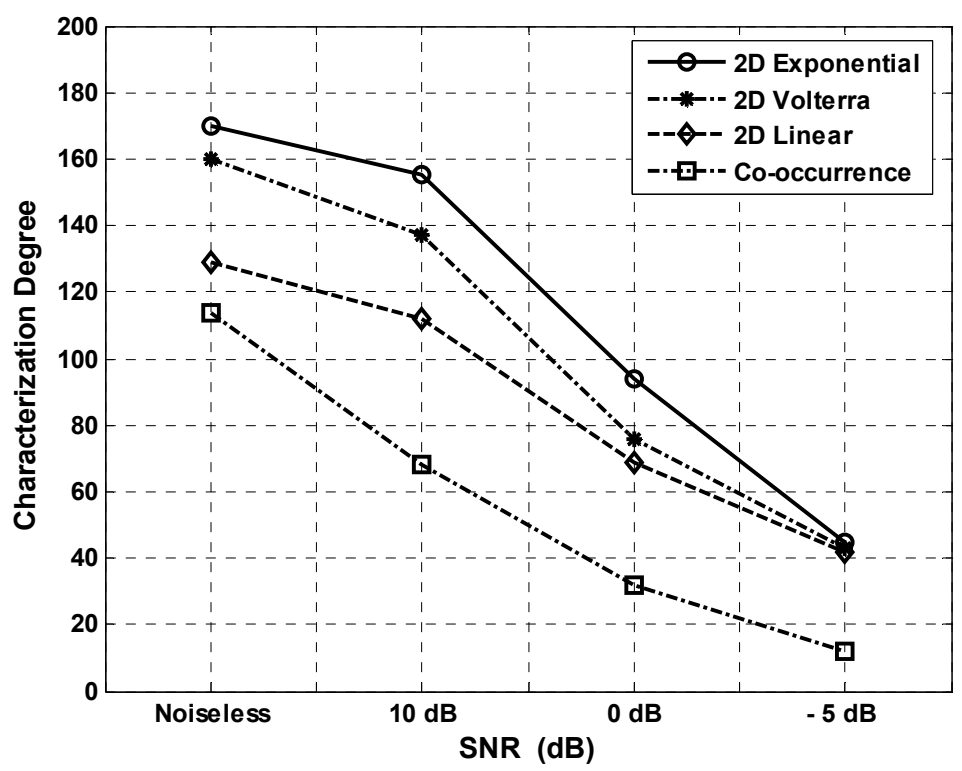

Figure 6: Plot of the characterization degree $C D$ of the 2-D adaptive exponential, Volterra and linear filters and the co-occurrence method for various SNR values (Filter order $9 \times 9$ ).

\section{Experiment 4: Comparison to the 2-D linear adaptive modeling based on higher order statistics}

In this experiment, we check whether the use of higher order statistics based algorithms improves the texture characterization in comparison to the already tested methods. For this reason, the lattice coefficients issued from 2-D Overdetermined Lattice Recursive Instrumental Variable filter (2-D OLRIV) algorithm proposed in [3][38], which is based on higher order statistics, are used as textural features. The characterization efficiency of these coefficients is compared to the one obtained with the exponential, Volterra and linear 2-D coefficients as well as the co-occurrence features. The studied methods are applied to the characterization of the texture images from the database of experiments 1,2 and 3 without any additive noise. A filter order of $9 \times 9$ is used. The classification accuracies and the characterization degrees of the obtained coefficients of the studied methods are presented in Table 4 . The results prove the superiority of the proposed 2-D exponential filter with respect to the high order statistics based one for texture characterization.

As we have concluded in [37], we think that although higher order statistics based filters have some advantages, and although textures have some non Gaussian properties, the use of an adaptive filter based on higher order statistics to estimate the 2-D lattice coefficients provides coefficients with a large variance and disturb the classification process. In other words, there is no improvement by using a higher order statistics based adaptive algorithm to characterize the texture model in comparison to the other studied methods.

\begin{tabular}{|c|c|c|}
\hline & Classification Accuracy & Characterization Degree \\
\hline 2-D Nonlinear exponential filter & $98.4 \%$ & 171 \\
\hline 2-D Second order Volterra Filter & $96.8 \%$ & 160 \\
\hline 2-D linear Filter & $93.8 \%$ & 129 \\
\hline Co-occurence based method & $91 \%$ & 114 \\
\hline $\begin{array}{l}\text { 2-D OLRIV filter based on higher order } \\
\text { statistics [3][37] }\end{array}$ & $88 \%$ & 93 \\
\hline
\end{tabular}

Table 4: Classification accuracies and characterization degrees of all the studied texture characterization methods (Noiseless case, Filter order $9 \times 9$ ) 


\section{Conclusion}

Taking into account the nonlinear character of the textured images, a new nonlinear exponential adaptive 2-D filter for texture characterization is proposed in this paper. The nonlinear filter coefficients are updated with the 2-D LMS algorithm and its characterization ability is compared to the one provided by both 2-D linear, second-order Volterra filters and the co-occurrence matrix method. The proposed exponential filter is simpler than the second-order Volterra filter and the number of the used coefficients stills approximately the same as in the linear filter. Extensive experiments show that the nonlinear exponential coefficients give better results in texture discrimination than those of both linear and Volterra coefficients, even in a noisy context. Further works taking into account the effect of the morphological aspect of the texture and its details orientation on the nonlinear parametric model still remain to be done. Also, the generalization to more natural color and multi-spectral textures classification and segmentation is an important perspective of the present work.

\section{References}

[1] U.A. Ahmad, K. Kidiyo, R. Joseph, "Texture features based on Fourier transform and Gabor filters: an empirical comparison", ICMV 2007, International Conference on Machine Vision, Islamabad, Pakistan, 1:67-72, Dec. 2007.

[2] P. Brodatz, "Textures: a photographic album for artist and designers", Dovers, New York, 1966.

[3] V. Buzenac, R. Settiner and M. Najim, "A new cumulant-based lattice algorithm for adaptive identification of non Gaussian bi-dimensional AR processes", Proc. of IEEE ICASSP 96, Atlanta, USA, pages 3033-3036, 7-10 May 1996.

[4] D. Charalampidis, "Texture Synthesis: Textons Revisited", IEEE Trans. on Image Processing, 15(3):777-787, March 2006.

[5] R. Chellappa and R.L. Kashyap, "Texture synthesis using 2-D non causal autoregressive models", IEEE Trans. On Acoustic, Speech and Signal Processing, 33(1):194-203, Feb. 1985.

[6] I. Claude, A. Smolarz, "A new textured image segmentation algorithm by auto-regressive modeling and multiscale block classification", Intern. Conf. on Image Processing and its Applications, 2:586-590, July 1997.

[7] D.A. Clausi, D. Huang, "Design-based texture feature fusion using Gabor filters and co-occurrence probabilities", IEEE Trans. on Image Processing, 14(7):925-936, July 2005.

[8] M. L. Comer, E. J. Delp, "Segmentation of textured images using a multiresolution Gaussian autoregressive model", IEEE Trans. on Image Processing, 8(3):408-420. March 1999.

[9] F. Fnaiech, M. Sayadi, and M. Najim, "Adaptive recursive higher order polynomial filter", NSIP'99, IEEE-EURASIP Workshop on Nonlinear Signal and Image Processing, Antalya, Turkey, 20-23 June 1999.

[10] J. M. Francos, A. Z. Meiri, B. Porat; "A wold-like decomposition of 2-D discrete homogeneous random fields", Ann. Appl. Prob., 5:248 - 260, 1995.

[11] J. M. Francos, "Cramér-Rao Bound on the Estimation Accuracy of Complex-Valued Homogeneous Gaussian Random Fields", IEEE Trans. on Signal Processing, 50(3):710-724, March 2002.

[12] H. Greenspans, S. Belongic, R. Goodman, "Rotation invariant texture recognition using a steerable pyramid", Proc. Of ICPR'94, International Conference on Pattern Recognition, 1:162-167, 1994.

[13] S.E. Grigorescu, N. Petkov, P. Kruizinga, "Comparison of texture features based on Gabor filters", IEEE Trans. on Image Processing, 11(10):1160-1167, Oct. 2002.

[14] M. Hadhoud, D W. Thomas. "The Two-dimensional adaptive LMS algorithm", IEEE Trans. on Circuits and Systems, 35(5):485-493, May 1988.

[15] M. Haindl, Unsupervised Texture Segmentation, Lecture Notes in Computer Science 1451, Springer, 1998.

[16] G. M. Haley and B. S. Manjunath, "Rotation-invariant texture classification using a complete space-frequency model", IEEE Trans. Image Processing, 8:255-269, February 1999.

[17] T. E. Hall and G. B. Giannakis, "Image modeling using inverse filtering criteria with application to textures", IEEE Trans. on Image Processing, 5(6):938-949, June 1996.

[18] R.M. Haralick, K. Shanmungan, and I. Dinstein, "Textural features for image classification", IEEE Trans. on Systems, Man, and Cybernetics, 3(6):610- 621, November 1973.

[19] F. V. Heijd, Image based measurement system, John Wiley and sons Edition, UK, 1994.

[20] J. Kook Kim and Hyun Wook Park, "Statistical Textural features for detection of Micro calcifications in Digitized Mammograms", IEEE Trans. on Medical Imaging, 18(3):231-238, March 1999.

[21] S. Krishnamachari and R. Chellappa, "Multiresolution Gauss-Markov Random Field Models for Texture Segmentation", IEEE Trans. on Image Processing, 6(2):251-267, July 1997.

[22] J. N. Lin and R.Unbehauen, "2-D adaptive CPWL Filter for 2-D nonlinear Channel equalization and Image Restoration", Electronics Letters, 28:6310-6318, 1992. 
[23] J. N. Lin and R.Unbehauen, "2-D adaptive Volterra filter for 2-D nonlinear channel equalization and image restoration", Electronics Letters, 28:180-182, 1992.

[24] X. Liu and M. Najim, "A two dimensional fast lattice recursive least squares algorithm", IEEE Trans. on Signal Processing, 44(10):2557-2567, Oct. 1996.

[25] S. Marcelja, "Mathematical description of the responses of simple cortical cells", J. Opt. Soc. Amer., (1)70:1297$1300,1980$.

[26] S. K. Mitra, H. Li, I-S. Lin and T. Yu, "A new class of nonlinear filters for image enhancement", IEEE ICASSP'91,Toronto, Canada, 4:2525-2529, 1991.

[27] N. Mittal, D. P. Mital, K.L. Chan, "Features for texture segmentation using Gabor filters", International Conference on Image Processing and its Applications, Dublin, Ireland, 1:353-357, Jul. 1999.

[28] J.A. Montoya-Zegarra, N.J. Leite, R. Torres, "Rotation-Invariant and Scale-Invariant Steerable Pyramid Decomposition for Texture Image Retrieval", Brazilian Symposium on Computer Graphics and Image Processing,(1):121-128, Oct. 2007.

[29] T. Ojala, M. Pietikäinen and D. Harwood, "A comparative study of texture measures with classification based on feature distributions", Pattern Recognition, 29(1):51-59, 1996.

[30] T. Ojala, M. Pietikäinen and J. Nisula, "Determining composition of grain mixtures by texture classification based on feature distributions", International Journal of Pattern Recognition and Artificial Intelligence, (10):73-82, 1996.

[31] T. Ojala, M. Pietikäinen, T. Mäenpää, "Multiresolution Gray-Scale and Rotation Invariant Texture Classification with Local Binary Patterns", IEEE Trans. on Pattern Analysis and Machine Intelligence, 24(7):971-987, July 2002.

[32] I. Pitas and A.N. Venetsanopoulos, Nonlinear digital filters, Principles and applications, Kluwer Academic Publishers, 1990.

[33] G. Ramponi and G. L. Sicuranza, "Quadrartic digital filters for image processing", IEEE Trans. on Acoustic, Speech and Signal Processing, 36(6):937-939, June 1988.

[34] T. Randen and J. H. Husoy, "Filtering for Texture Classification: A Comparative Study", IEEE Trans. on Pattern Analysis and Machine Intelligence, 21(4):291-310, Apr. 1999.

[35] G. Rellier, X. Descombes, F. Falzon, J. Zerubia, "Texture feature analysis using a Gauss-Markov model in hyperspectral image classification", IEEE Trans. Geosciences and Remote Sensing, 42(7):1543-1551, 2004.

[36] A. Sarkar, K. M. S. Sharma, and R. V. Sonak, "A New Approach for Subset 2-D AR Model Identification for Describing Textures", IEEE Trans. on Image Processing, 6(3):407-413, March 1997.

[37] M. Sayadi and M. Najim, "Comparison of second and third order statistics based adaptive filters for texture characterization", IEEE ICASSP 99, Arizona, USA, 6:3281-3284, March 1999.

[38] M. Sayadi, V. Buzenac and M. Najim, "Texture characterization using 2-D cumulant-based lattice adaptive filtering", IEEE ICASSP 98, Seattle, USA, 3:2725-2728, May 1998.

[39] M. Sayadi, S. Sakrani, F. Fnaiech and M. Cheriet, "A new nonlinear exponential 2-D adaptive filter and its application in texture characterization" IEEE ICASSP'2004, Montreal, Canada, USA, 17-21 Mai 2004.

[40] M. Sayadi, F. Fnaiech and M. Najim, "An LMS adaptive second-order Volterra filter with a zeroth-order term: steady state performance analysis in a time-varying environment", IEEE Trans. on Signal Processing, 47(3):872-876, March 1999.

[41] G. Tzagkarakis, B. Beferull-Lozano, P. Tsakalides, "Rotation-Invariant Texture Retrieval with Gaussianized Steerable Pyramids", IEEE Trans. on Image Processing, 15(9):2702-2718, 2006.

[42] G. Van de Wouwer, P. Scheunders, and D. V. Dyck, "Statistical Texture Characterization from Discrete Wavelet Representations", IEEE Trans. on Image Processing, 8:592-598, 1999.

[43] Y. Wang, X. Wei, S. Xiao, "LBP Texture Analysis Based on the Local Adaptive Niblack Algorithm", CISP, Congress on Image and Signal Processing, Hainan, China, (2):777-780, May 2008.

[44] Q. Xu, J. Yang, S. Ding, "Texture Segmentation using LBP embedded Region Competition", Electronic Letters on Computer Vision and Image Analysis, 5(1):41-47, 2005.

[45] Y. Zhao, L. Zhang, P. Li, and B. Huang, "Classification of High Spatial Resolution Imagery using Improved Gaussian Markov Random-Field-Based Texture Features", IEEE Trans. Geosciences and Remote Sensing, 45(5):14581468, May 2007. 